\title{
Effect of Hydrogen Exposure on the Notch Tensile Properties of High Strength Steel in Hydrogen Gas Environment*
}

\author{
Yuichi SUZUKI**, Hisatake ITOGA*** and Hiroshi NOGUCHI***,**** \\ ** Graduate School of Engineering, Kyushu University \\ ${ }^{* * *}$ National Institute of Advanced Industrial Science and Technology (AIST) \\ ${ }^{* * \star \star}$ Department of Mechanical Engineering, Faculty of Engineering, \\ Kyushu University, 744 Moto-oka, Nishi-ku, Fukuoka-shi, Fukuoka, 819-0395 Japan \\ E-mail: nogu@mech.kyushu-u.ac.jp
}

\begin{abstract}
The effect of hydrogen exposure on high strength steel, SCM435, based on a sharp notched specimen was investigated. Tensile tests were carried out in hydrogen gas and helium gas environments. In the hydrogen gas environment, specimens that were not exposed to hydrogen gas and specimens that were exposed to hydrogen gas for $48 \mathrm{~h}$ were used. As the result of the tensile tests with specimens of various notch depths, the tensile strengths of the hydrogen-gas-exposed specimens were higher than that of the non-exposed specimens. The tensile strength increases with the exposure time in hydrogen gas until hydrogen homogeneously diffuses in the entire specimen for the notched specimens of $t=1.0 \mathrm{~mm}$. The fracture surfaces of the specimens in hydrogen gas showed an intergranular fracture near the notch root while the specimens in helium gas showed a fracture mode of microvoid coalescence. The crack initiation stress of the non-exposed specimen was lower than that of the 48h-hydrogen-exposed specimen.
\end{abstract}

Key words: Fracture, Hydrogen, Tensile Strength, High Strength Steel, Notch, Hydrogen Gas Exposure, Hydrogen Distribution, Intergranular Fracture, TDS

\section{Introduction}

Recently, hydrogen is expected to be a new energy. For example, fuel cell vehicles and hydrogen vehicles are being developed, and pipe-lines and storage tanks for supplying hydrogen are made of metal. The hydrogen storage tank for $35 \mathrm{MPa}$ hydrogen in the FCV is filled with $40 \mathrm{MPa}$ hydrogen. The $900 \mathrm{MPa}$ class SCM435 steel is used for the tank. Because of the need to increase the mileage of these vehicles, a tank for 70MPa-hydrogen is being developed. For filling high-pressure gas and for weight saving, use of a higher strength metal is expected. However, it is well known that high strength steel is sensitive to hydrogen, a delayed fracture occurs and the hydrogen embrittlement sensitivity is high $^{(1),(2)}$. Because it is necessary to clarify the effect of hydrogen when using high strength steel, there have been some studies ${ }^{(3),(4)}$ of the effect on the static strength or the mechanism. It is known that the entire concentration of hydrogen in the specimen can be measured by Thermal Desorption Spectroscopy (TDS), and the tensile strength and the fatigue strength are degraded by the hydrogen content. For example, M. Wang et al. ${ }^{(5)}$ clarified that the tensile strength of both a plain and notched specimen of 1,450 MPa class AISI4135 steel decreased with the hydrogen content due to hydrogen electric charge. Y.D. Li et al. ${ }^{(6)}$ also reported that the fatigue strength of the long-life region of high strength spring steel

*Received 9 May, 2011 (No. T2-09-0245) Japanese Original : Trans. Jpn. Soc. Mech. Eng., Vol.75, No.758, A (2009), pp.1300-1308 (Received 23 Mar., 2009) [DOI: 10.1299/jmmp.6.265]

Copyright () 2012 by JSME 
decreased with the hydrogen content due to hydrogen gas charge at high pressure and high temperature. In previous studies, although there have been some examinations on the effect of homogeneously diffused hydrogen in a specimen due to the hydrogen charge or hydrogen gas, there is no report on the effect of the distributed hydrogen. Although it is necessary to evaluate the effects of hydrogen content and hydrogen distribution on the strength because the hydrogen in the specimen changes with time due to hydrogen pressure and the temperature, these effects have not been clarified.

Therefore, in this study, a tensile test of a 1,300 MPa class SCM435 specimen with a circumferential notch was carried out in hydrogen gas and helium gas. Moreover, a hydrogen-exposed and non-exposed specimen were used, and the effect of the hydrogen exposure on their strength was studied.

\section{Specimens and experimental procedure}

\subsection{Specimen}

The material used was a quench-tempered chromium-molybdenum steel, SCM435.

Table 1 and Fig.1 show the chemical composition and the specimen configuration, respectively. The specimen has a minimum section diameter of $d=6.0 \mathrm{~mm}$, a notch depth of $t=0.02 \sim 1.0 \mathrm{~mm}$ and a notch root radius of $\rho=0.03 \mathrm{~mm}$. The surface of the plain specimen and the notch root of the notched specimen were buff-polished with $0.05 \mu \mathrm{m}$ alumina powder so that the roughness of the surfaces would not influence the experimental results.

Table 1 Chemical composition (mass \%).

\begin{tabular}{ccccccccc}
\hline $\mathrm{C}$ & $\mathrm{Si}$ & $\mathrm{Mn}$ & $\mathrm{P}$ & $\mathrm{S}$ & $\mathrm{Ni}$ & $\mathrm{Cr}$ & $\mathrm{Mo}$ & $\mathrm{Cu}$ \\
\hline 0.38 & 0.22 & 0.84 & 0.024 & 0.021 & 0.08 & 1.11 & 0.15 & 0.12 \\
\hline
\end{tabular}

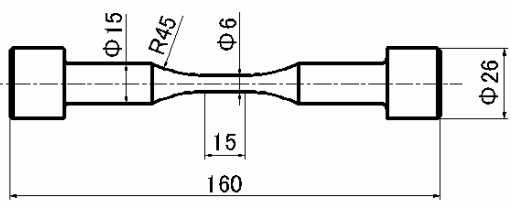

(a) Plain specimen

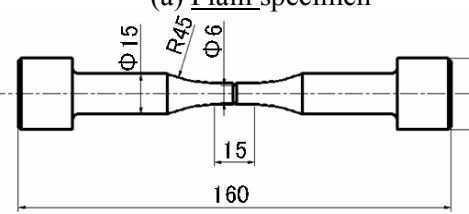

(b) Notched specimen

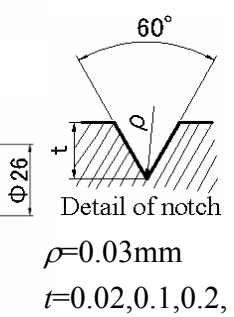

$0.5,1.0 \mathrm{~mm}$

Fig.1 Specimen configuration.

\subsection{Experimental procedure}

Tensile tests were carried out in helium gas or hydrogen gas. The test conditions included a gas pressure of $0.6 \mathrm{MPa}$, room temperature of $20 \sim 25{ }^{\circ} \mathrm{C}$ and a cross-head speed of $0.000675 \mathrm{~mm} / \mathrm{s}$. For the non-exposed specimen, the tensile test started just after filling the gas in the chamber, while for the xh-H-exposed specimen the test started after exposing the specimen to hydrogen gas for $\mathrm{x} h$.

Various notch depths, $t=0.02 \sim 1.0 \mathrm{~mm}$, were adopted, and the tests were carried out in helium gas and hydrogen gas in order to study the effect of the notch depth on the notch tensile properties in hydrogen gas. The non-exposed specimen was tested in helium gas, while the non-exposed and the 48h-H-exposed specimens were tested in hydrogen gas. Moreover, in order to study the effect of the 
exposure time, the exposure time was changed between 0 and $48 \mathrm{~h}$ for the specimen with a notch depth of $t=1.0 \mathrm{~mm}$ in hydrogen gas.

\subsection{Hydrogen content measurement}

The hydrogen content was measured by Thermal Desorption Spectroscopy (TDS) for determining the hydrogen exposure time. The condition of the H-exposed-specimen is that the hydrogen sufficiently enters the center of the specimen.

\subsubsection{Theoretical hydrogen content}

In an infinitely long cylinder with the radius $r_{0}$ from the initial condition of the surface with hydrogen content of $C_{0}$, the hydrogen content of $C$ at the distance $r$ from the center after time $t$ is obtained ${ }^{(7) \sim(8)}$ by Fick's second law for a constant temperature diffusion.

$$
C / C_{0}=1-2 \sum_{n=1}^{\infty} \frac{\exp \left(\frac{-D \beta_{n}^{2} t}{r_{0}^{2}}\right) J_{0}\left(\frac{\beta_{n} r}{r_{0}}\right)}{\beta_{n} J_{1}\left(\beta_{n}\right)}
$$

Here, $D\left[\mathrm{~m}^{2} / \mathrm{s}\right]$ is the hydrogen diffusion coefficient $\left(D=1.0 \times 10^{-10} \mathrm{~m}^{2} / \mathrm{s}\right.$ for SCM435 $\left.{ }^{(9)}\right), J_{0}$ and $J_{1}$ are the first kind of the 0 -th and 1st Bessel functions, respectively, and $\beta_{\mathrm{n}}$ is the root of the first kind of the 0 -th Bessel function: $J_{0}(\beta)=0$. Figure 2 (a) shows the theoretical diffusion time obtained from Eq (1) at the cylindrical center $(r=0)$ with the diameter of $d^{\prime}=6 \mathrm{~mm}$. The ordinate is the relative hydrogen content $C / C_{0}$ and the abscissa is the hydrogen diffusion time. The cylindrical diameter $d$ ' corresponds to the minimum section diameter of Fig. 1 (b). As shown in Fig. 2 (a), the relative hydrogen content $C / C_{0}$ at the center is saturated when the hydrogen diffusion time is about 30 hours. Next, denoting the average hydrogen content at time $t$ as $C_{\text {ave, }}$, the relative average hydrogen content is obtained as follows from Eq. (1).

$$
C_{\text {ave }} / C_{0}=1-4 \sum_{n=1}^{\infty} \frac{\exp \left(\frac{-D \beta_{n}^{2} t}{r_{0}^{2}}\right)}{\beta_{n}{ }^{2}}
$$

Figure 2 (b) shows the values from Eq. (2). The ordinate is the relative average hydrogen content $C_{\text {ave }} / C_{0}$ and the abscissa is the hydrogen diffusion time. Similar to the result of Eq. (1), the hydrogen content in the entire specimen volume is saturated when the diffusion time is about $30 \mathrm{~h}$.

\subsubsection{Measured results}

Because the hydrogen content measured by TDS is the mass content of the total hydrogen in the specimen, the measured value can be compared to Fig. 2(b). Figure 3 shows the H-exposed specimen and the measured specimen by TDS. The specimen was exposed to $0.6 \mathrm{MPa}$ hydrogen at room temperature and exposure times of $4 \mathrm{~h}, 6 \mathrm{~h}, 48 \mathrm{~h}$ and $96 \mathrm{~h}$, which were similar to the conditions for the tensile specimen. After the exposure, a sample with a thickness of $t=2.0 \mathrm{~mm}$ was cut from the specimen center, and the hydrogen content was measured by TDS at a heating rate of $0.5{ }^{\circ} \mathrm{C} / \mathrm{s}$ to $800{ }^{\circ} \mathrm{C}$. Figure 4 shows the results. The ordinate is the hydrogen content and the abscissa is the hydrogen exposure time. Because the hydrogen content is about $0.25 \mathrm{ppm}$ after the exposure time of 6 $\mathrm{h}$, it is considered that the hydrogen is saturated. However, the exposure time of $6 \mathrm{~h}$ is shorter than the theoretical saturated time in Fig. 2 (b). Therefore, $48 \mathrm{~h}$ is adopted as the saturated exposure time in this study. 


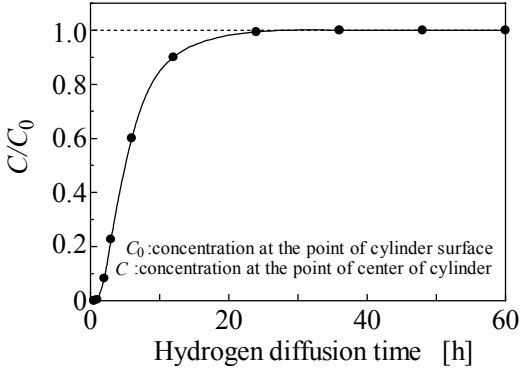

(a) Hydrogen concentration at center of infinite cylinder of diameter $d^{\prime}=6 \mathrm{~mm}$.

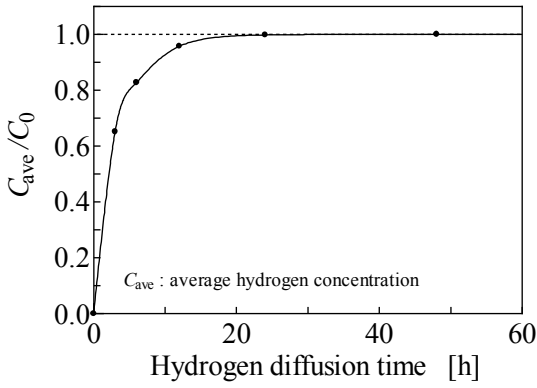

(b) Hydrogen concentration of infinite cylinder of diameter $d^{\prime}=6 \mathrm{~mm}$

Fig.2 Theoretical curves of hydrogen concentration.

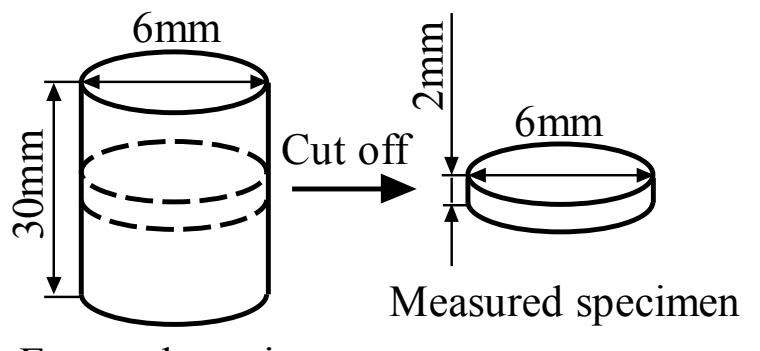

\section{Exposed specimen}

Fig.3 Exposed specimen and measurement sample configuration.

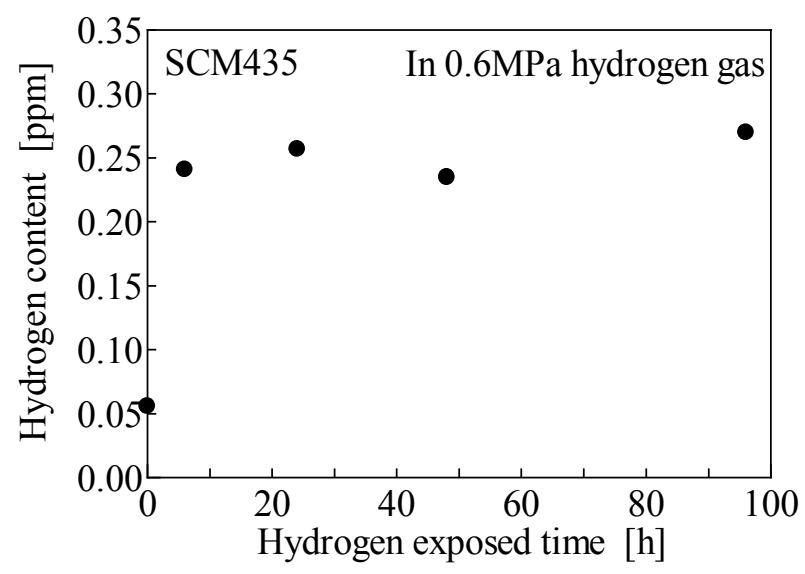

Fig.4 Relationship between hydrogen content and exposure time.

\section{Experimental results and discussion}

\subsection{Effect of hydrogen exposure on notch strength}

Figure 5 shows the stress-elongation curves of a specimen in helium gas, a non-exposed specimen and an H-exposed specimen in hydrogen gas. In the plain specimen, the tensile strength in hydrogen gas is equal to that in helium gas, and they rupture after the necking occurs. There are no difference in both the fracture-elongation and reduction-of-area in the helium gas and hydrogen gas. The fracture-elongation and reduction-of-area of the notched specimen decrease in both the helium gas and hydrogen gas. The fracture-elongation and the reduction-of-area decrease with the notch depth for a specimen with a notch deeper than $t=1.0 \mathrm{~mm}$ in helium gas and that deeper than $t=0.2$ $\mathrm{mm}$ in hydrogen breaks without a large deformation. Figure 6 shows the relationship between the 
tensile strength and the notch depth. The tensile strengths of a specimen in helium gas and a 48h-H-exposed specimen in hydrogen gas increase with the notch depth, but that of a non-exposed specimen decreases. The reason why the tensile strength increases with the notch depth in helium gas is due to the fact that the plastic constraint becomes severer under the tri-axial stress condition based on the notch shape. The notched specimen with the highest tensile strength is the specimen in helium gas followed by the H-exposed specimen in hydrogen gas. The non-exposed specimen in hydrogen gas has the least tensile strength. The differences among these specimens are greater with the notch depth. This is based on the fact that the effect of the hydrogen gas on the tensile strength increases with the notch depth.

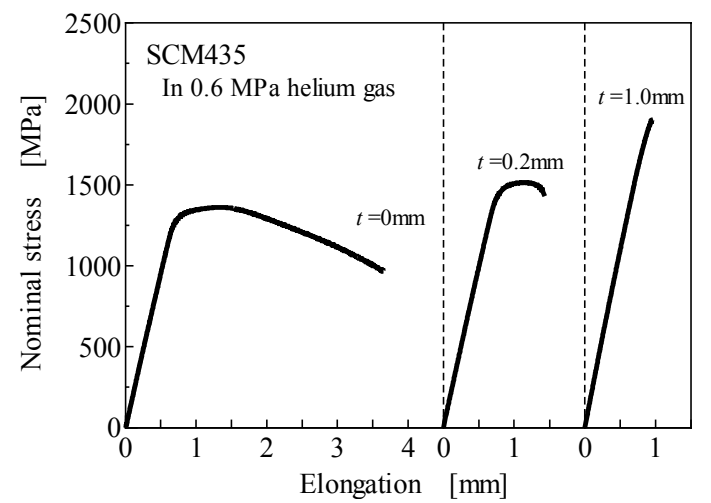

(a) In helium gas

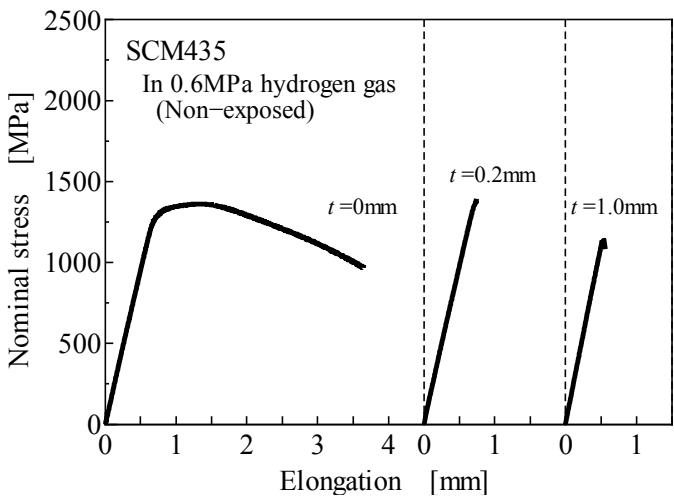

(b) In hydrogen gas (non-exposed)

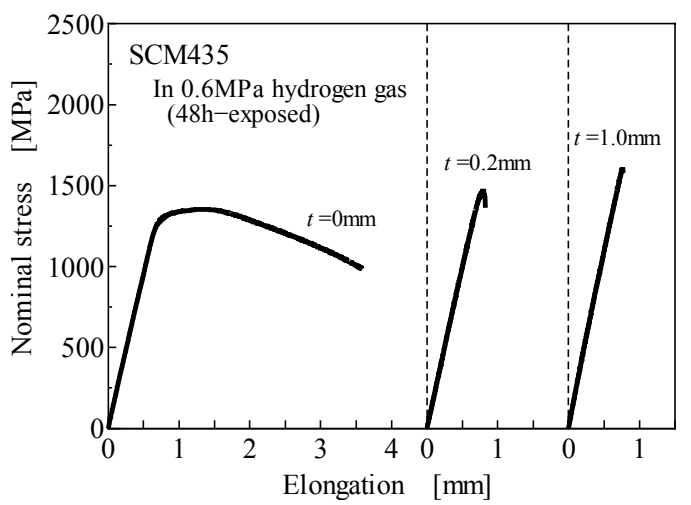

(c) In hydrogen gas (48h-H-exposed)

Fig.5 Relationship between nominal stress and elongation. 


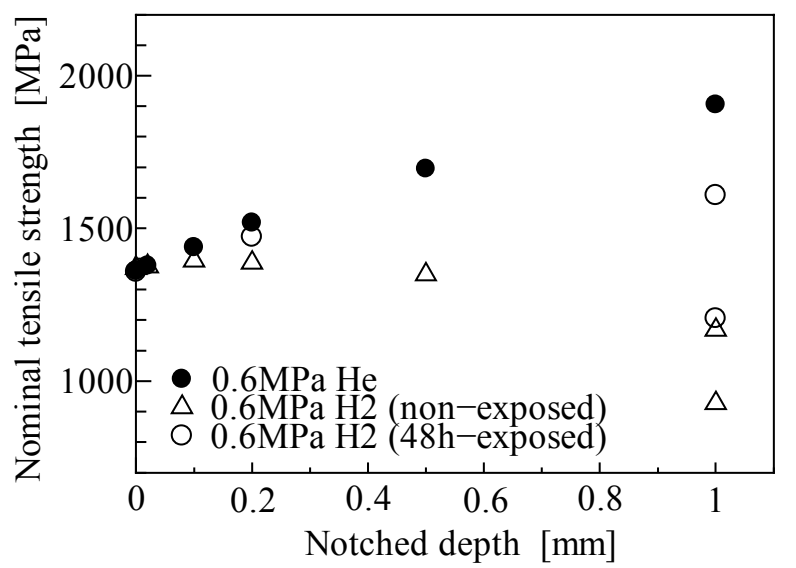

Fig.6 Relationship between tensile strength and notched depth.

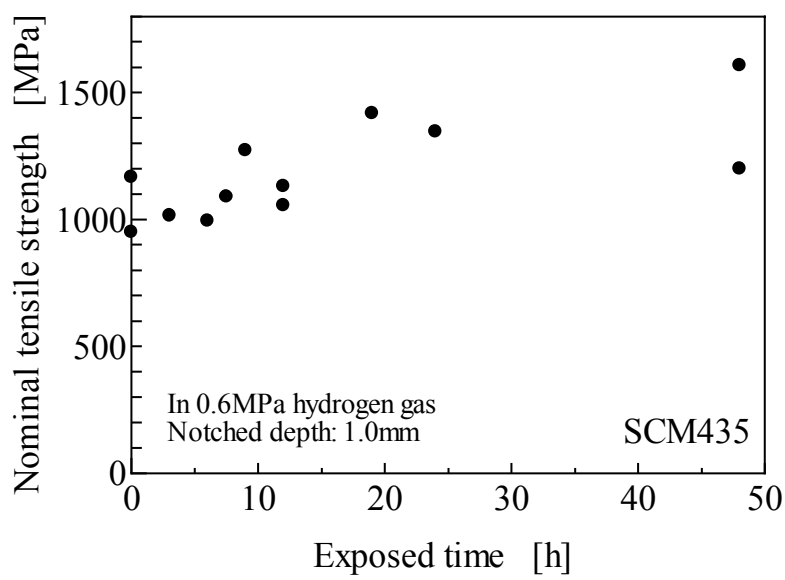

Fig.7 Relationship between tensile strength and hydrogen exposure time.

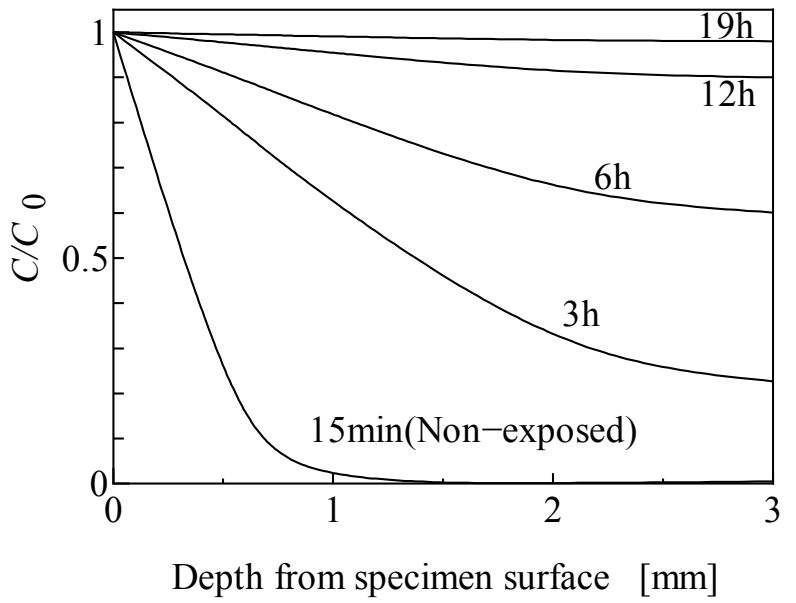

Fig.8 Relationship between hydrogen concentration and depth from specimen surface.

\subsection{Effect of exposure time in hydrogen gas}

Figure 7 shows the relationship between the tensile strength and the exposure time. The tensile strength increases with the exposure time between $0 \mathrm{~h}$ and $19 \mathrm{~h}$, but slightly decreases between $19 \mathrm{~h}$ and $48 \mathrm{~h}$. 
When the exposure time is changed, the hydrogen content and the hydrogen content distribution are changed. The total amount of hydrogen in the specimen increases with the exposure time as shown in Fig. 2 (b). Because M. Wang et al. ${ }^{(5)}$ pointed out that the tensile strength of a notched high tensile strength steel decreased with the electric charge time, it is thought that the increase in the mount of hydrogen decreases the tensile strength. However, the present study shows that the tensile strength increases with the amount of hydrogen. Therefore, in order to consider the effect of the hydrogen content and the hydrogen content distribution, we showed the nominal hydrogen content distribution for each hydrogen exposure time calculated from Eq. (1) in Fig. 8. The abscissa shows the depth from the specimen surface, and the ordinate shows the relative hydrogen content at the point from the specimen surface. As shown in Fig. 8, the hydrogen content in the center of the specimen is low, hense there is a content difference between the center and the surface when the exposure time is short. Therefore, the hydrogen at an internal point increases with time, and the relative hydrogen content becomes uniform in the entire specimen after the saturated exposure time. Because the hydrogen content at the specimen surface is constant and independent of the exposure time, the total amount of hydrogen depends on the hydrogen content in the internal volume of the specimen as shown in Fig. 2 (b). In the notched specimen, the tensile strength is influenced by the hydrogen content at the notch root and around the root, which are the fracture origins. Because M. Wang et al. ${ }^{(5)}$ changed the total amount of hydrogen by electric charge, the hydrogen content at the notch changed. However, in this study, the hydrogen content at the notch root of the $\mathrm{H}$-exposed specimen is independent of the exposure time, and it is then speculated that the hydrogen content distribution near the notch root is a cause of the decrease in the tensile strength. The gradient of hydrogen content distribution becomes smaller with the hydrogen exposure time, and there is no gradient of hydrogen content distribution after the exposure time of 20 $\mathrm{h}$. The tensile strength increases at the exposure times of $0 \mathrm{~h} \sim 19 \mathrm{~h}$, and it is saturated with the exposure times of $19 \mathrm{~h} \sim 48 \mathrm{~h}$. The tendency of the change in tensile strength corresponds to that of the decrease in hydrogen content distribution, and it can be inferred that the higher gradient of hydrogen content distribution decreases the tensile strength. However, because Fig. 8 shows the nominal hydrogen content distribution with no consideration of the stress gradient and plastic constraint due to the notch before the tensile test, the above discussion is based on the initial condition of the specimen.

\subsection{Fracture surface observation}

The fracture surface was observed by a scanning electron microscope (SEM). In the plain specimens under all conditions, a cup-and-cone fracture occurs as shown in Figs. 9 (a), (b) and (c), and it fractures from the specimen center. The fracture surface is covered with a ductile fracture surface of dimples as shown in Fig. 9 (d), and there is no difference between that in hydrogen gas and that in helium gas.

On the other hand, in the notched specimen, the fracture occurred from the notch root or in the vicinity except for the specimen of $t=0.02 \mathrm{~mm}$ in the helium gas in which the cup-and-cone fracture occurred. Although the specimen macroscopically broke perpendicular to the loading direction, there was a macroscopic shear fracture part in the final breaking part in hydrogen gas. Figures 10,11 and 12 show the fracture surfaces of the specimen with a notch depth of $t=1.0 \mathrm{~mm}$ in helium gas, the non-exposed specimen in hydrogen gas and 48h-H-exposed specimen in hydrogen gas, respectively. In helium gas, the fracture surface is divided into two regions; one is the striped pattern region parallel to the notch root as shown in Fig. 10 (b) and the other is inside the one. A dimple fracture is observed in the region of (b) (Fig. 10 (c), the dimple and facet are observed in the inside region (Fig. 10 (d)). The crack propagates from all the surroundings of the notch root to the center of the specimen and results to the fracture. In helium gas, similar fracture surfaces are observed in the other notch depths. 


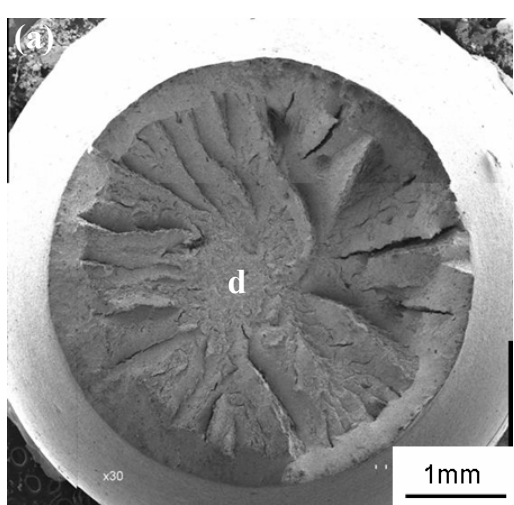

(a) In helium gas

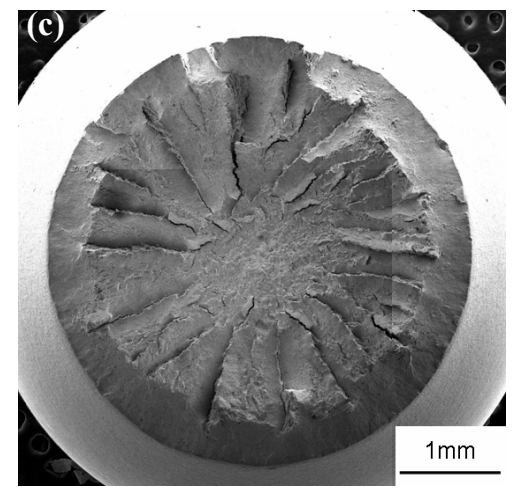

(c) In hydrogen gas (48h-H-exposed)

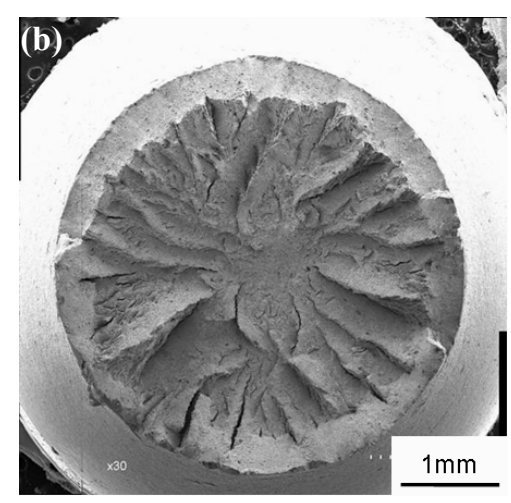

(b) In hydrogen gas(non-exposed)

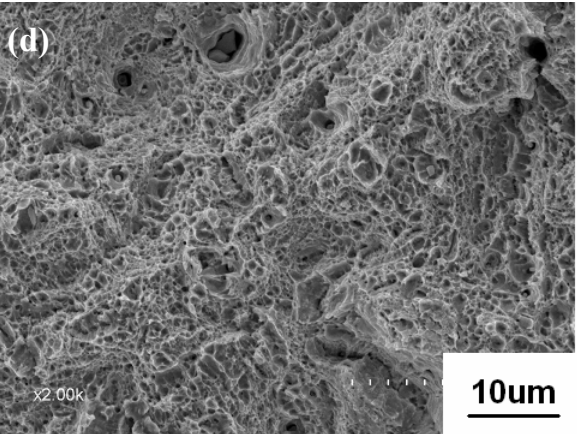

(d) Microvoid of (a)

Fig.9 Fracture surfaces of smooth specimens.

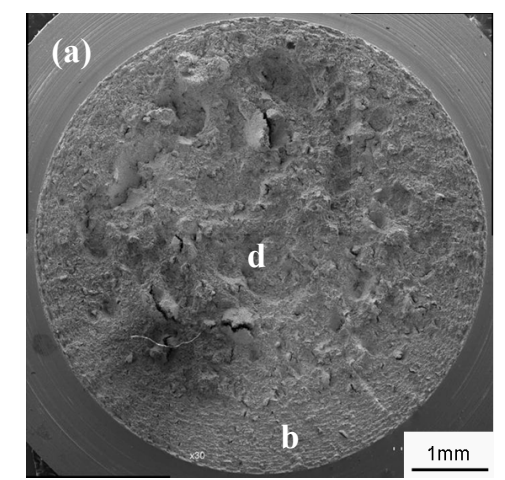

(a) General view at low magnification

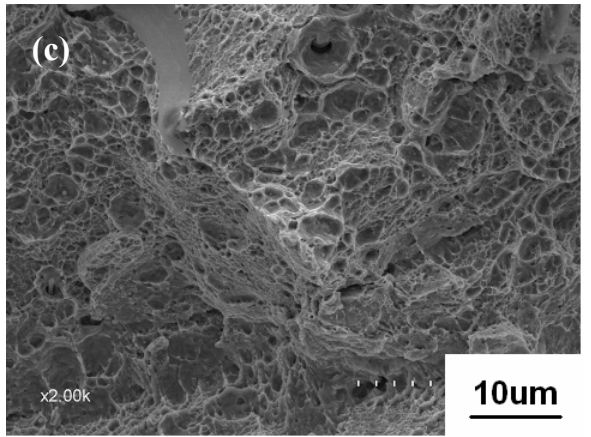

(c) Microvoid near the notch root of (b)

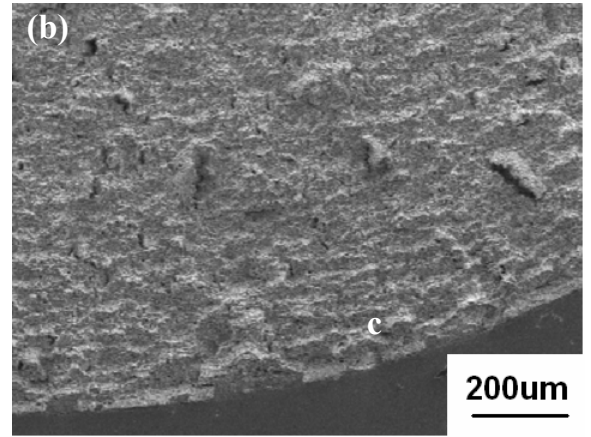

(b) Ductile fracture at crack initiation site of (a)

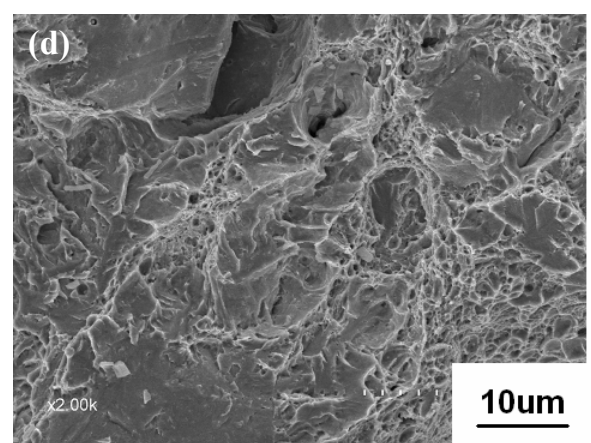

(d) Microvoid and facets of (a)

Fig.10 SEM fractographs of notched specimen with $t=1.0 \mathrm{~mm}$ in helium gas. 


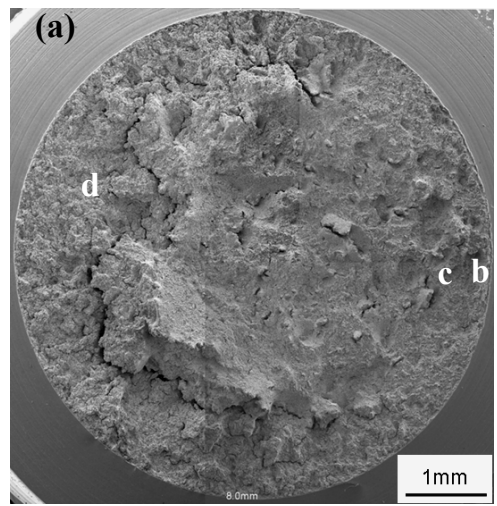

(a) General view at low magnification

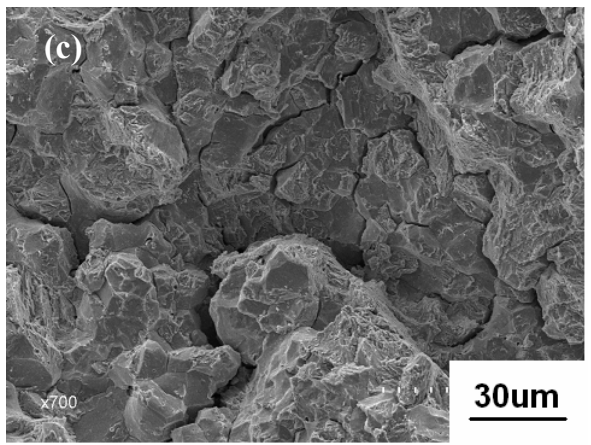

(c) Intergranular of (a)

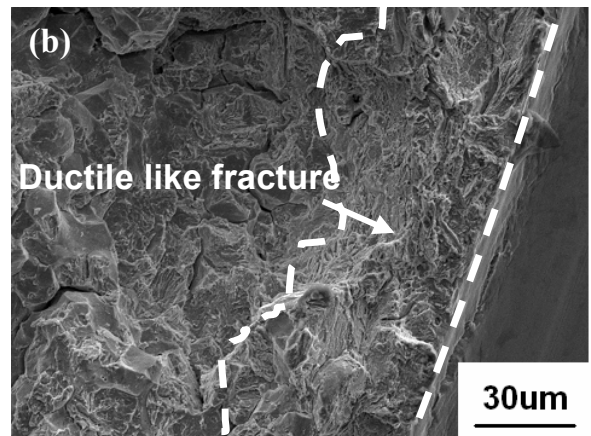

(b) Ductile fracture near the notch root of (a)

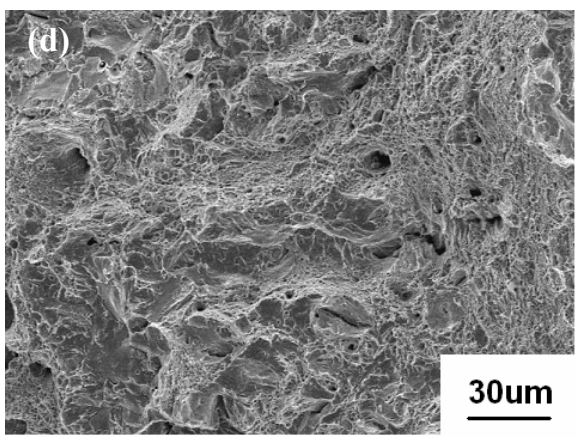

(d) Microvoid and facets of (a)

Fig.11 SEM fractographs of non-exposed specimen with $t=1.0 \mathrm{~mm}$ in hydrogen gas.

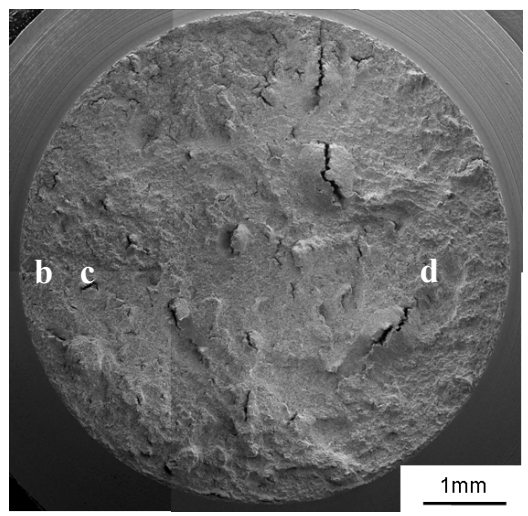

(a) General view at low magnification

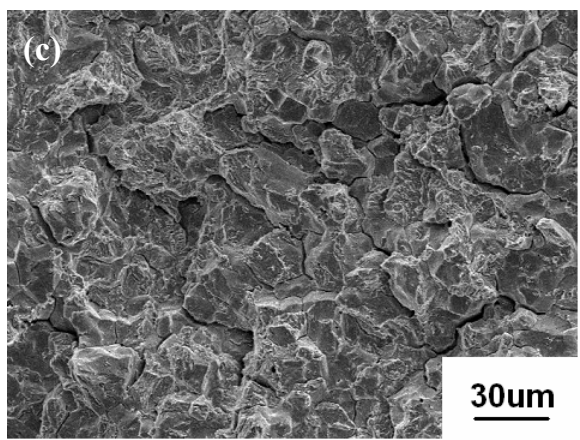

(c) Intergranular of (a)

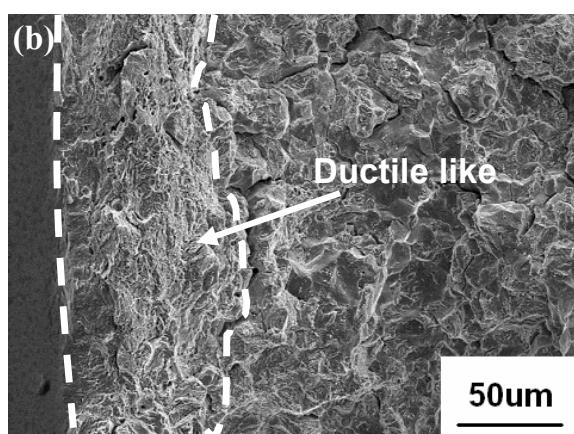

(b) Ductile fracture near the notch root of (a)

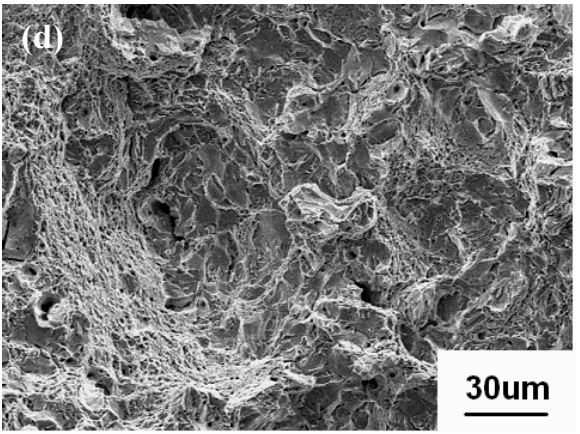

(d) Microvoid and facet of (a)

Fig.12 SEM fractographs of $48 \mathrm{~h}-\mathrm{H}$-exposed specimen with $t=1.0 \mathrm{~mm}$ in hydrogen gas. 
In hydrogen gas, there are a ductile-like fracture surface near the notch root (Fig. 11(b) and Fig. 12(b)), the intergranular fracture surface inside of it (Figs. 11(c) and 12(c)) and the dimple fracture surface and facet (Figs. 11(d) and 12(d)). There are no differences in the fracture surfaces of the non-exposed specimen and the $48 \mathrm{~h}-\mathrm{H}$-exposed specimen. Although the crack propagates in the specimen in helium gas from all surroundings of the notch root to the center of the specimen and the fracture surface is axis-symmetric, that in hydrogen is not the same. The intergranular fracture surface is observed near the notch root, and there is an intergranular fracture surface with a dimple fracture surface on the boundary of the macroscopic shear fracture. Therefore, the fracture process in the hydrogen gas can be considered as follows. 1 . With the hydrogen concentrated at the notch root, the crack due to the slip or the grain boundary crack occurs near the notch root. 2 . The hydrogen is concentrated at the nucleated crack, thus crack can propagate. The crack nucleated from one point or some points and macroscopically propagates in one direction. 3. The unstable final fracture is caused by the macroscopic shear stress.

The macroscopic shear fracture path was observed in most of the fracture surfaces except for some cases as shown in Fig. 12 (a). It is speculated that the tensile strength degradation in hydrogen gas is caused by the differences in the crack nucleation and crack propagation in the hydrogen gas and in helium gas. Similar fracture surfaces are also observed for the other notch depths. However, the intergranular fracture surfaces increase and the dimple fracture surfaces decrease when the notch depth is deeper and the tensile strength is lower.

\subsection{Crack observation at the notch root}

There are a ductile fracture surface near the notch root, an intergranular fracture surface, a dimple fracture surface and the unstable fracture paths under the all hydrogen gas conditions, while there are no particular differences in all the fracture morphologies. Therefore, the differences in strengths with exposure time is considered based on the difference in the surface crack that occurred on the surface of the plain specimen. Figure 13 shows the crack that was nucleated on the broken surfaces of the plain specimens under the non-exposed and $48 \mathrm{~h}-\mathrm{H}$-exposed conditions. Although there is no surface crack in the 48h-H-exposed specimen, many surface cracks are observed on the specimen surface near the fracture surface of the non-exposed specimen. Because there are many cracks on the specimen surface near the fracture surface, a crack is nucleated in a region in which the plastic deformation becomes larger after necking. Because plastic deformation occurs at the notch root of a notched specimen, it is thought that a similar difference in the crack nucleation occurs in the notch root. It is speculated that the tensile strength of the non-exposed specimen is lower than that of the $48 \mathrm{~h}-\mathrm{H}$-exposed specimen, because the crack at the notch root can occur more easily. Therefore, in order to study the difference in the crack nucleation of the notched specimen, using the non-exposed specimen and the $48 \mathrm{~h}-\mathrm{H}$-exposed specimen, the notch root was observed just before the final fracture. Using specimens with $t=1.0 \mathrm{~mm}$ in which there is the largest difference in the tensile strengths, the specimens were interrupted at the stress level shown in Fig. 14, and the cracks were observed. Test A and Test B are $95 \%$ as large as the tensile strength of the non-exposed specimen and $48 \mathrm{~h}-\mathrm{H}$-exposed one, respectively, and Test $\mathrm{C}$ is $75 \%$ as large as the tensile strength of the $48 \mathrm{~h}-\mathrm{H}$-exposed specimen (or $95 \%$ as large as the tensile strength of the non-exposed specimen). Figure 15 shows the notch root after the tests. There was a long crack in the Test A specimen, and the crack existed in about half of the surroundings. There were many small cracks in the Test B specimen in all the surroundings of the notch root, while there was no crack in the Test $\mathrm{C}$ specimen except for some extremely small cracks. It is found from these observed results that the crack nucleation stress level of the non-exposed specimen is lower than that of the $48 \mathrm{~h}-\mathrm{H}$-exposed specimen, and the tensile strength difference due to the exposure time is based on the crack nucleation difference. It can be deduced from the relationship between the hydrogen content distribution due to the hydrogen exposure time and the tensile strength that the exposure affects the hydrogen content distribution, and the distribution influences the crack nucleation, and then it changes the tensile strength. 
Moreover, because a crack was observed at a notch root as a semicircle in hydrogen gas, it suggests that a crack does not uniformly propagate from all the surroundings of the notch root, but that it propagates in a direction from a point.
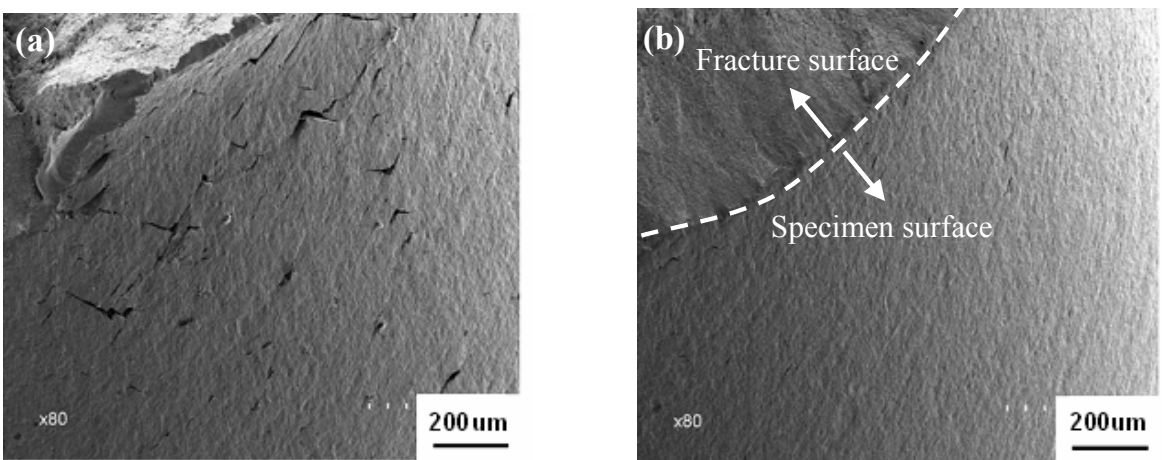

Fig.13 The surface of smooth specimen near the fracture surface: (a)in hydrogen gas(non-exposed specimen),(b)in hydrogen gas(exposed specimen).

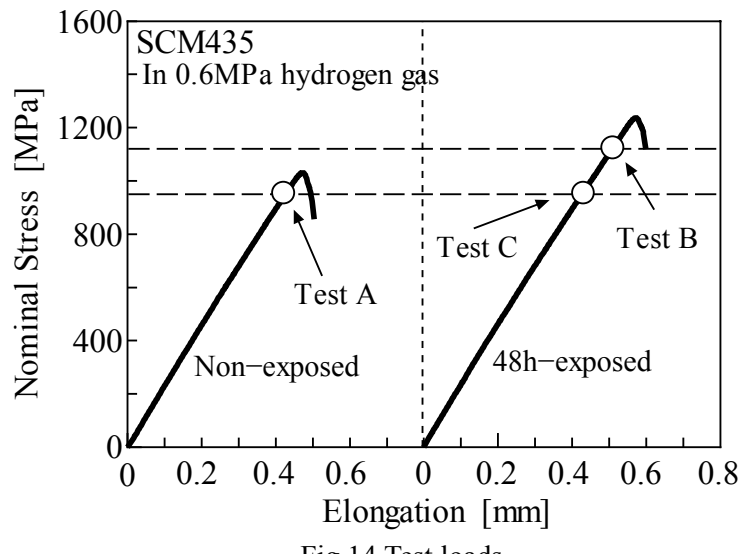

Fig.14 Test loads.

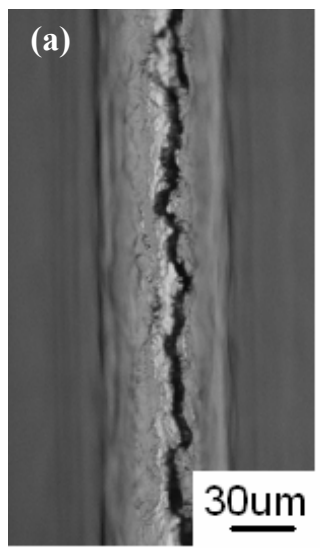

(a) Test A

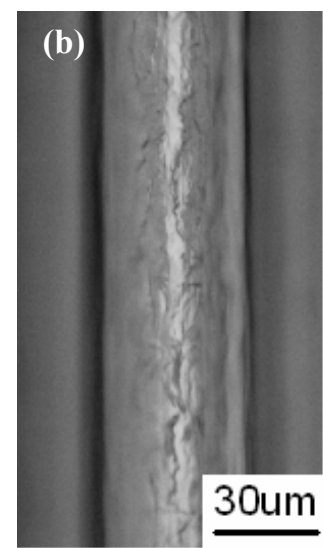

(b) Test B

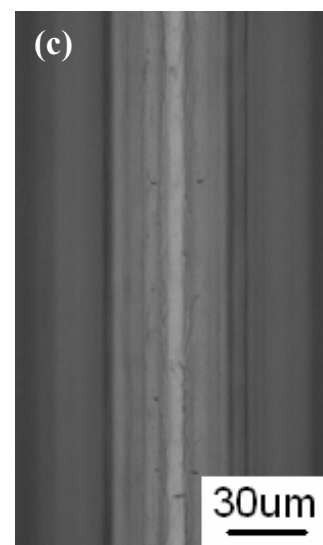

(c) Test C

Fig. 15 Cracks in the notch root of Fig. 14.

\subsection{Discussion}

In this study, the effect of the gradient of hydrogen content distribution on the tensile strength is macroscopically considered. The results suggested that the tensile strength decreases due to the gradient of hydrogen content distribution. It is necessary in the next step to microscopically analyze it based on the microfracture mechanism in the stress gradient and the plastic deformation due to the 
notch. Moreover, it is necessary to evaluate the effect of the hydrogen content and gradient of hydrogen content distribution under various conditions in order to use the high strength steel in a hydrogen gas environment. Because the crack initiation limit at the notch root depended on the H-exposure time, it is necessary to observe the fracture process for clarifying the mechanism. Clarifying the mechanism leads to using the present experimental data for predicting the tensile strength under other conditions.

Therefore, the next study is to investigate the H-exposure effect on the tensile strength at various hydrogen pressures, test temperatures, strain rates and notch root radii. The effect of the hydrogen content and the gradient of hydrogen content distribution will also be examined in detail. Moreover, it is necessary to consider the degradation mechanism of the tensile strength due to hydrogen based on the fracture process observation.

\section{Conclusions}

Tensile tests of the high strength steel SCM435 with the circumferential notch were carried out in helium gas and hydrogen gas, and the fracture surface and the crack at the notch root were observed. The following results were then obtained.

1) The tensile strength of the plain specimen in hydrogen gas is the same as that in helium gas. However, there is a difference in the strengths of notched specimens, and the difference increases with the notch depth.

2) The tensile strength of the $24 \mathrm{~h}-\mathrm{H}$-exposed specimen with a notch is greater than that of the non-exposed specimen.

3) The tensile strength of the notched specimen depends on the H-exposure time. It is speculated that the phenomenon is caused by the hydrogen content distribution.

4) The crack initiation limit of the non-exposed specimen is lower than that of the $48 \mathrm{~h}-\mathrm{H}$-exposed specimen.

\section{Acknowledgement}

The major part of this study was conducted as part of the "Fundamental Research Project on Advance Hydrogen Science" funded by the New Energy and Industrial Technology Development Organization (NEDO).

\section{References}

(1) Matsuyama S., in: Delayed Fracture, The Nikkan Kogyo Shimbun, LTD., Tokyo ,(1989).

(2) Fukai Y., Tanaka K. and Uchida H., Hydrogen and Metal, Uchida Rokakuho, Tokyo ,(1998).

(3) Matsuoka S., Homma N., Tanaka H., Fukushima Y. and Murakami Y., Effect of Hydrogen on the Tensile Properties of 900-MPa-Class JIS-SCM435 Low-Alloy-Steel for Use in Storage Cylinder of Hydrogen Station, The Japan Institute of Metals, Vol.70, No.12 (2006), pp. 1002-1011.

(4) Chang T.L., Tsay L.W., Chen C., Influence of gaseous hydrogen on the notched tensile strength of D6ac steel ,Material Science and Engineering, A316 (2001), pp.153-160.

(5) Wang M., Akiyama E., Tsuzaki K., Effect of hydrogen on the fracture behavior of high steel during slow strain rate test, Corrosion Science 49 (2007), pp.4081-4097.

(6) Li Y.D., Yang Z.G., Liu Y.B., Li S.X., Li G.Y., Hui W.J., Weng Y.O., The influence of hydrogen on very high cycle fatigue properties of high strength spring steel ,Material Science and Engineering A 489 (2008), pp. 373-379

(7) Demaret A., Hock A.G., Meunier F.A., Diffusion of hydrogen in mild steel, Acta 
Metallurgia, Vol.2, (1954) 3 pp214.

(8) Fujii T., Nomura K., Temperature Dependence of Hydrogen Diffusivity of 21/4Cr-1Mo Steel, The iron and Steel Institute of Japan 70-1 (1984), pp. 104-111.

(9) Nagata J., Guy N. and Murakami Y., Effect of hydrogen charge on fatigue strength of martensitic stainless steel, Journal of the Society of Materials Science, Japan, Vol. 54 No.12 (2005), pp.1217-1224. 